\title{
Glomerular filtration rate in children and young adults with haemato-oncological disease and infection is best described by three-compartmental iohexol model
}

\author{
Hiie Soeorg ${ }^{1}$, Aveli Noortoots ${ }^{1}$, Maarja Karu², Kadri Saks², Jana Lass ${ }^{3}$, Irja Lutsar ${ }^{1}$, and \\ Lenne-Triin Kõrgvee ${ }^{3}$ \\ ${ }^{1}$ University of Tartu \\ ${ }^{2}$ Tallinn Children's Hospital \\ ${ }^{3}$ Tartu University Hospital
}

February 8, 2021

\begin{abstract}
Background. Children with cancer and infection may develop glomerular hyperfiltration (GH). With the aim to determine the prevalence of $\mathrm{GH}$ in children and young adults with haemato-oncological disease and infection we developed population pharmacokinetic model of iohexol. We further aimed to assess the accuracy of estimated glomerular filtration rate (eGFR) equations and single- or two-point measured GFR (mGFR) formulas compared with GFR based on iohexol clearance from our population pharmacokinetic model (iGFR). Procedure. Hospitalized patients (0.5-25 years) with haemato-oncological disease and infection were included if their eGFR was [?] $80 \mathrm{~mL} / \mathrm{min} / 1.73 \mathrm{~m} 2$ at the screening visit. Iohexol plasma concentrations were described by population pharmacokinetic model. Bias, precision and accuracy of 23 eGFR equations and 18 mGFR formulas were calculated. Results. Total of 32 iohexol administrations was performed in 28 patients. Median (range) eGFR was 136 (74-234) $\mathrm{mL} / \mathrm{min} / 1.73 \mathrm{~m} 2$ and age 15.1 (0.8-26.0) years. Three-compartment model with allometric scaling of central, one peripheral compartment and clearance (with power 0.75) to weight fitted the best. Median (range) iGFR was 103 (68-140) $\mathrm{mL} / \mathrm{min} / 1.73 \mathrm{~m} 2$. All except one eGFR equation overestimated GFR. Lund-Malmö revised eGFR equation performed the best, followed by Gao equation. Of single- or two-point mGFR formulas 15 overestimated iGFR. Modified Jacobsson formula at 5.5 $\mathrm{h}$ performed the best, followed by Fleming formula at $3 \mathrm{~h}$. Conclusions. In children and young adults with haemato-oncological disease and infection renal function is best described by iohexol clearance from three-compartmental pharmacokinetic model, while eGFR equations and single- and two-point mGFR formulas overestimate iGFR.
\end{abstract}

\section{Introduction}

Glomerular hyperfiltration (GH), conventionally defined as glomerular filtration rate (GFR) larger than mean +/- two standard deviations of healthy individuals ${ }^{1}$, has been detected in 10-50\% of children with cancer $^{2-5}$. The reason for GH could be tumor breakdown resulting in increased solute load to the kidneys and hypermetabolic state ${ }^{4,5}$. Concomitant bacterial infection and its treatment may even further increase $\mathrm{GFR}^{6}$

Determining the prevalence of $\mathrm{GH}$ requires correct measurement of GFR, but the predictive performance of commonly used creatinine-based equations for calculating estimated GFR (eGFR) performs poorly in children with cancer ${ }^{2,3,7}$. Use of biomarkers like cystatin $\mathrm{C}^{8,9}$ and $\beta_{2}$-microglobulin (B2MG) ${ }^{10,11}$ is 
possible alternative for estimating GFR, but these may introduce bias, because inflammation increases the concentration of both markers ${ }^{8,10}$ and B2MG is further elevated due to malignancies ${ }^{10}$.

For accurate assessment of GFR, measured GFR (mGFR) is increasingly determined using exogeneous markers, of which iohexol with its advantages of low cost, stability and low interlaboratory variation, is considered one of the best ${ }^{12}$, and formulas based on biexponential decline ${ }^{13}$. As two-compartment models may overestimate mGFR ${ }^{14}$ compared to three-compartmental population pharmacokinetic models that often fit better to iohexol plasma concentration data ${ }^{14-16}$, the prevalence of $\mathrm{GH}$ in children with cancer may have been overestimated.

With the aim to describe renal function in children and young adults with haemato-oncological disease and infection requiring antibacterial treatment we developed iohexol population pharmacokinetic model. We additionally aimed to describe the accuracy of creatinine-, cystatin C- or B2MG-based eGFR equations and single- or two-point mGFR formulas and to determine the prevalence of GH based on iohexol clearance according to the final population pharmacokinetic model in this population.

\section{Methods}

\section{Study population}

Two prospective clinical trials were performed from 15.01.2016 to 30.06.2019 assessing the possible effect of GFR on pharmacokinetics of cefepime (EudraCT 2015-000631-32) and piperacillin-tazobactam (EudraCT 2016-003374-40) at the Department of Haematology and Bone Marrow Transplant of Tartu University Hospital and Department of Haematology and Oncology of Tallinn Children's Hospital. Patients aged from 6 months to 25 years were eligible if they 1) had malignant or benign haematological or oncological disease (in piperacillin-tazobactam trial) or malignant tumor (in cefepime trial), 2) had received immunosuppressive or chemotherapy within one year prior to the study, 3) currently received antibacterial treatment with piperacillin-tazobactam or cefepime on clinical indications, 4) had central venous catheter, peripheral venous or arterial catheter in place, 5) had eGFR [?] $80 \mathrm{ml} / \mathrm{min} / 1,73 \mathrm{~m}^{2}$ (according to Schwartz equation in children $(<19$ years) or CKD-EPI equation in adults ([?]19 years)) at the screening visit. Informed consent was obtained from legal guardian of the child and assent also asked from minors older than 6 years. The study was approved by the Research Ethics Committee of the University of Tartu.

\section{Iohexol administration and plasma concentration measurement}

Iohexol was administered as an intravenous injection of $2 \mathrm{~mL}$ (if patient's weight was $<40 \mathrm{~kg}$ ) or $5 \mathrm{~mL}$ (weight $>40 \mathrm{~kg}$ ) of Omnipaque (GE Healthcare AS)? corresponding to 1294 or $3235 \mathrm{mg}$ of iohexol (300 mg iodine/ml), respectively. Blood samples were collected prior to and $5 \mathrm{~min}, 30 \mathrm{~min}, 3 \mathrm{~h}$ and $5.5 \mathrm{~h}$ or $7.5 \mathrm{~h}$ after administration of iohexol and centrifugated within $15 \mathrm{~min}$ of sampling, or, if not possible, refrigerated at $+4^{\circ} \mathrm{C}$ for up to $2 \mathrm{~h}$ and centrifuged thereafter. Plasma samples were stored at $-80{ }^{\circ} \mathrm{C}$ until iohexol concentration measurements by ultra-performance liquid chromatography-tandem mass spectrometer at the Analytical Services International Ltd (St George's - University of London, London, UK). Additional blood sample was drawn $3 \mathrm{~h}$ after administration of iohexol to measure plasma concentrations of creatinine, cystatin $\mathrm{C}$ and B2MG. Creatinine was measured by enzymatic assay and cystatin $\mathrm{C}$ and B2MG by immunoturbidimetric assay according to manufacturers' instructions.

\section{Monitoring of patients}

Patients were monitored for possible adverse events up to 6 days after administration of iohexol. Physiological parameters (weight, height, blood pressure, respiratory and cardiac rate, blood oxygen saturation, body 
temperature), diagnoses, laboratory parameters taken on clinical indications and concomitant medications used within 6 days from the start of antibacterial treatment were recorded.

\section{Variables}

Body surface area (BSA) was calculated according to Mosteller formula $\left(\mathrm{BSA}=\right.$ weight $(\mathrm{kg})^{0.5} \times$ height $(\mathrm{cm})^{0.5} / 60$ ). For children, z-score of body mass index (weight $(\mathrm{kg}) /$ height $(\mathrm{m})^{2}$ ) for age was calculated according to World Health Organization growth references ${ }^{17,18}$.

eGFR was calculated by creatinine-based equations (Schwartz ${ }^{19}$, Full Age Spectrum equation based on age $\left(\mathrm{FAS}_{\text {age }}\right)$ or height $\left(\mathrm{FAS}_{\text {height }}\right)^{20}$, Lund-Malmö revised ${ }^{21}$, $\mathrm{Gao}^{22}$, Millisor ${ }^{23}$, Brandt $^{24}$ and Cole equations using weight $\left(\mathrm{Cole}_{\text {weight }}\right)$ or weight and creatinine $\left(\mathrm{Cole}_{\mathrm{Cr}}\right){ }^{25}$ for all patients; Chronic Kidney Disease Epidemiology Collaboration equation (CKD-EPI) ${ }^{26}$, Modification of Diet in Renal Disease equation (MDRD) 27. Cockcroft-Gault equation adjusted for body surface area (CG) ${ }^{28}$ for adults only), cystatin C-based equations (Schwartz $\left(\mathrm{Schwartz}_{\mathrm{Cys}}\right)^{29,30}$, Full Age Spectrum $\left(\mathrm{FAS}_{\mathrm{Cys}}\right)^{31}$, Caucasian Asian Pediatric Adult $\left(\mathrm{CAPA}_{\mathrm{CysC}}\right)^{32}$, Berg $^{33}$, CKD-EPI $\left.\left(\mathrm{CKD}-\mathrm{EPI}_{\mathrm{CysC}}\right)^{34}\right)$, creatinine- and cystatin C-based equations (Schwartz $\left(\text { Schwartz }_{\mathrm{Cr}-\mathrm{CysC}}\right)^{29,30}$, Full Age Spectrum combining $\mathrm{FAS}_{\mathrm{cys}}$ and $\mathrm{FAS}_{\text {age }}\left(\mathrm{FAS}_{\text {age-CysC }}\right)$ or $\mathrm{FAS}_{\text {cys }}$ and $\mathrm{FAS}_{\text {height }}\left(\mathrm{FAS}_{\text {height-CysC }}{ }^{31}\right.$, CKD-EPI (CKD-EPI Cr-CysC ${ }^{34}$ ) and B2MG-based equations (Ikezumi ${ }^{35}$ for children, CKD-EPI (CKD-EPI B2MG $_{\text {}}$ ) for adults ${ }^{36}$ ). Expressions of all eGFR equations are in Supplementary Material.

mGFR based on iohexol concentrations at single time points $(3 \mathrm{~h}$ or $5.5 \mathrm{~h}$ ) was calculated using the following formulas (expressions in Supplementary Material): Fleming, Ham and Piepsz, Groth and Aasted, Stake, Jacobsson and modified (mod.) Jacobsson formula. mGFR based on iohexol concentrations at two time points $(3 \mathrm{~h}$ and $5.5 \mathrm{~h}$ ) was calculated using slope-intercept method and the following corrections were used (expressions in Supplementary Material): Brøchner-Mortensen for adults $\left(\mathrm{BM}_{\text {adult }}\right)$ or children $\left(\mathrm{BM}_{\text {child }}\right)$, Fleming, Jødal and Brøchner-Mortensen (JBM), BM child $_{\text {modified by Schwartz }}\left(\mathrm{BM}_{\text {Schwartz }}\right)$, JBM modified by $\mathrm{Ng}\left(\mathrm{JBM}_{\mathrm{Ng}}\right)$. Clearance estimate was normalized to BSA before applying correction to mGFR ${ }^{37}$.

GH was defined as GFR larger than mean plus two standard deviations of GFR in healthy individuals ${ }^{1}$. For children aged 6-8, 8-12, 12-18, 18-24 months the thresholds were 100, 118, 128, $131 \mathrm{~mL} / \mathrm{min} / 1.73 \mathrm{~m}^{2}$, respectively ${ }^{38}$. For children $>2$ years and young adults, the threshold was $150 \mathrm{~mL} / \mathrm{min} / 1.73 \mathrm{~m}^{2}$, as the mean of various studies in these age groups ${ }^{13}$.

\section{Pharmacokinetic analysis}

Plasma concentrations of iohexol were described by nonlinear mixed-effects models in software NONMEM (version 7.4). One-, two-, three-compartmental models were tested for basic structural plasma pharmacokinetic model. Concentrations below lower limit of quantification (3 $\mathrm{mg} / \mathrm{L}$ for piperacillin/tazobactam group, $17 \mathrm{mg} / \mathrm{L}$ for cefepime group) were handled by M3 method ${ }^{39}$. Interindividual variability was assumed to be log-normally distributed and was considered for all parameters. Of covariates total body weight was tested and retained in the model if it decreased objective function value. Allometric scaling to $70 \mathrm{~kg}$ were tested for all parameters; for clearance allometric exponent 0.75 and for volumes of distribution allometric exponent 1 were tested. Proportional, additive, and combined error models were tested. Goodness-of-fit plots and visual predictive check were used to assess the fit of model. GFR based on individual estimates of iohexol clearance $(\mathrm{L} / \mathrm{h})(\mathrm{iGFR})$ from final model was calculated as iGFR $\left(\mathrm{mL} / \mathrm{min} / 1.73 \mathrm{~m}^{2}\right)=$ clearance $(\mathrm{L} / \mathrm{h}) / 60 \times 1000$ $/ \mathrm{BSA}\left(\mathrm{m}^{2}\right) \times 1.73$.

\section{Statistical analysis}

Statistical analysis was performed in software R (version 3.6.1). The performance of eGFR equations and single- or two-point mGFR formulas to estimate iGFR was assessed in terms of bias by median difference 
between iGFR and eGFR or mGFR (iGFR-eGFR, iGFR-mGFR), precision by interquartile range of differences, accuracy by median absolute difference and the percentage of eGFR or mGFR within $\pm 10 \%$ (P10) or $\pm 30 \%$ (P30) of iGFR. Bland-Altman plots (iGFR as the reference method on the x-axis ${ }^{40}$, difference between iGFR and eGFR or mGFR (iGFR-eGFR, iGFR-mGFR) on the y-axis) show $95 \%$ limits of agreement (mean \pm two standard deviations of difference).

\section{Results}

\section{Study population}

Twenty-eight patients were included in the study of whom 11 (39\%) had diagnosis of solid tumors, 10 (36\%) lymphoma, 7 (25\%) leukemia or other hematological disease (one non-malignant hematological disease). Four patients were included twice in the study as permitted by the protocol, with median (range) time difference 73 (50-459) days and median (range) weight change 11 (1-26) kg between iohexol administration occasions and were considered as separate cases in analysis. Therefore, results from total of 32 iohexol administrations were included in the analysis. Demographic and clinical characteristics of study patients are shown in Table 1.

\section{Iohexol population pharmacokinetic model}

Total of 177 plasma concentrations of iohexol were obtained. Three-compartmental model with allometric scaling of central and one peripheral compartment and clearance (with power 0.75) to weight provided the best fit to the data. Interindividual variability was retained only in clearance. Residual variability was described by proportional error. Estimates of the parameters of the final pharmacokinetic model are shown in Table 2. Goodness-of-fit plots (Figure 1, Supplementary Figure 1) and visual predictive check (Supplementary Figure 2) showed good fit of the final model to data.

According to iGFR from the final population pharmacokinetic model no-one had GH, median (range) iGFR was $103(68-140) \mathrm{mL} / \mathrm{min} / 1.73 \mathrm{~m}^{2}$. Two patients had adverse events possibly related to iohexol: non-itching macular rash on arms immediately after iohexol administration; skin rash $24 \mathrm{~h}$ after iohexol administration (managed with antihistamines and one dose of oral prednisolone), although the exact etiology remains unclear as the rash appeared also approximately $48 \mathrm{~h}$ after the first ever dose of piperacillin-tazobactam.

\section{Performance of eGFR equations and mGFR formulas}

The performance of eGFR equations and mGFR formulas to estimate iGFR is shown in Table 3 and Table 4. Bland-Altman plots of eGFR equations and mGFR formulas are shown in Supplementary Figure 3 and 4. From the performance assessment, a patient with eGFR $914 \mathrm{~mL} / \mathrm{min} / 1.73 \mathrm{~m}^{2}$ according to Schwartz equation was omitted as an outlier.

All eGFR equations overestimated iGFR, except creatinine-based Millisor equation and B2MG-based equations (Table 3). Overall, LM equation performed the best in terms of precision and accuracy and the secondbest in terms of bias. It was followed by Gao equation that was in terms of precision and accuracy the second- (P10) or third-best (IQR, MAE, P30), Schwartz ${ }_{\mathrm{Cr}-\mathrm{CysC}}$ and Schwartz $\mathrm{CysC}_{\mathrm{s}}$ equations that had the second-best MAE or P30, respectively, and Ikezumi equation with the third-best P10.

In all 31 iohexol administrations (excluding the case with eGFR value of $914 \mathrm{~mL} / \mathrm{min} / 1.73 \mathrm{~m}^{2}$ as outlier), plasma concentrations were determined at $3 \mathrm{~h}$ (median (range) time of sampling 180 (162-192) min after iohexol administration) and in 14 cases at $5.5 \mathrm{~h}$ (median 328 (286-334) min after iohexol administration). All mGFR formulas overestimated iGFR, except Fleming formula at $3 \mathrm{~h}$ and Groth and Aasted formula at 3 or $5.5 \mathrm{~h}$ (Table 4). Overall, mod. Jacobsson formula at $5.5 \mathrm{~h}$ performed the best in terms of accuracy (MAE, P10, P30) and the second-best in terms of precision and bias. It was followed by Fleming formula at $3 \mathrm{~h}$ 
being the best in bias and the second-best in terms of accuracy (MAE, P10) and $\mathrm{BM}_{\text {child }}$ two-point formula that was the third-best in terms of bias and accuracy (MAE, P10).

\section{Discussion}

To the best of our knowledge, this is the first study assessing GFR by iohexol population pharmacokinetic model with the aim to describe the prevalence of GH in children and young adults with haemato-oncological disease and infection. Despite high eGFR values based on eGFR equations routinely used in clinical practice, none of the patients had GH according to iGFR. Three-compartmental model described iohexol pharmacokinetics the best, suggesting that the use of single- or two-point mGFR formulas based on biexponential decline of iohexol plasma concentration may not accurately estimate GFR in this population.

The absence of GH in our study population contrasts with previously found estimates of $10-50 \%{ }^{2-5}$. Possible explanation to such discrepancy is that we determined the prevalence using conventional definition of GH based on GFR estimates mostly established by single or multiple point mGFR formulas ${ }^{38}$, but not threecompartmental population pharmacokinetic model. For example, in the reference work for normal GFR values for children, GFR was determined by Ham and Piepsz formula at $120 \mathrm{~min}^{38}$, but the prevalence of GH in our study based on this formula at $3 \mathrm{~h}$ was $26 \%$, in line with other studies in children with cancer ${ }^{2-5}$. Such discrepancy in the prevalence estimates of GH based on iGFR and Ham and Piepsz formula reflects our finding that mGFR formulas overestimate iGFR. This is in agreement with a recent study showing that two-compartmental model overestimated iohexol clearance compared with three-compartmental model ${ }^{14}$. Although two-compartmental model is often chosen as the final model of iohexol concentration data ${ }^{41-43}$, three-compartmental models nevertheless provide better fit ${ }^{42}$, are not even tested ${ }^{43}$ or are chosen as often as two-compartmental models ${ }^{14-16}$. As plasma clearance of most is better described with at least threecompartmental model ${ }^{13}$, we believe that further studies assessing GFR by three-compartmental models are warranted to determine the true prevalence of $\mathrm{GH}$.

In line with other studies ${ }^{3,7,9}$, we found that most creatinine- and cystatin C-based eGFR equations overestimated iGFR, were imprecise and had low accuracy. In our study creatinine-based Lund-Malmö revised and Gao equation with small bias and good precision and large accuracy were the best performing equations, followed by cystatin C-based or combined (creatinine- and cystatin C-based) eGFR equations. This seemingly contrasts with other studies in children with malignancies that have shown better performance of cystatin C-based or combined (creatinine- and cystatin C-based) compared with solely creatinine-based eGFR equations ${ }^{2,9}$. However, to the best of our knowledge, previous studies in children with malignancies have not evaluated Lund-Malmö revised equation and Gao equation has been studied only once ${ }^{7}$. In a validation study in children with cancer ${ }^{7}$, Gao equation was the worst performing equation, but the study included younger children with lower renal function than in the equation development dataset on whom Gao equation performed the worse ${ }^{22}$. Recent large validation studies in European children showed that Lund-Malmö revised equation and Gao equation performed the best among creatinine-based eGFR equations in children with normal renal function (GFR [?] $75 \mathrm{~mL} / \mathrm{min} / 1.73 \mathrm{~m}^{2}$ ) ${ }^{44,45}$, that is with renal function comparable to that of our study patients. Still, in our study only in half of cases eGFR using these two equations was within $10 \%$ of iGFR, showing that these equations are nevertheless unsuitable for children with haemato-oncological disease and normal renal function. As pediatric equations are applicable to young adults as well ${ }^{20,46}$, we believe that inclusion of young adults to our cohort did not compromise their performance.

In large studies evaluating various eGFR equations, cystatin-C based, such as Schwartz ${ }_{\mathrm{Cr}-\mathrm{CysC}}$, and B2MGbased eGFR equations outperform most creatinine-based equations, including Lund-Malmo revised or Gao equation ${ }^{11,44,45}$. Poorer performance of $\mathrm{Sch} \mathrm{Sartz}_{\mathrm{Cr}-\mathrm{CysC}}$ compared with creatinine-based Lund-Malmo revised equation in our study could result from increased level of cystatin $\mathrm{C}$ due to inflammation ${ }^{8}$ in case of infection. Although B2MG-based Ikezumi eGFR equation showed the third-best P10 value and slightly better precision compared with Schwartz $\mathrm{Cr}_{\mathrm{Cr}-\mathrm{CySC}}$ equation, overall it was not superior to Lund-Malmo revised, Gao, Schwartz $\mathrm{Cr}_{\mathrm{CysC}}$ or $\mathrm{Schwartz}_{\mathrm{CysC}}$ equations. Such worse performance was somewhat expected 
because malignancies and inflammation increase the serum levels of B2MG ${ }^{10}$ that is probably reason for B2MG-based eGFR equations being the only iGFR-underestimating ones. It should be also noted that eGFR equations specifically developed based on children having malignancies (Brandt, Millisor, Cole weight $_{\text {, }}$ Cole $_{\mathrm{Cr}}$ ) were not among the best performing equations, similarly to a recent study ${ }^{7}$. The reason for their poor performance could be small dataset ${ }^{25}$, use of iothalamate that exhibits significant tubular secretion ${ }^{13}$ for development of the equation ${ }^{24}$ or exclusion of often incorporated covariates, such as age and sex, from the equation ${ }^{23}$, in contrast to Lund-Malmo revised and Gao equations ${ }^{21,22}$.

Due to many factors influencing the level of endogenous markers, it was expected that the eGFR equations based on endogenous markers perform notably worse than single- or two-point formulas of mGFR using iohexol concentrations. However, the performance of mGFR formulas was still poor. Most of these formulas overestimated iGFR, although we cannot exclude that the performance of two-point formulas could have been compromised by the use of samples drawn at 3 and $5.5 \mathrm{~h}$ instead of at 2 and $5 \mathrm{~h}$, when these formulas perform better ${ }^{37}$. The best performing formulas were single-point formulas - Fleming formula at $3 \mathrm{~h}$ and modified Jacobsson formula at $5.5 \mathrm{~h}$ - that were also the best performing ones in a recent study in children, despite modified Jacobsson formula was evaluated at $5 \mathrm{~h}{ }^{47}$, suggesting that these could be indeed well performing formulas. However, even these best performing formulas in our study had low precision and accuracy (Fleming single-point formula at $3 \mathrm{~h}$ ) or large bias (modified Jacobsson single-point formula at $5.5 \mathrm{~h}$ ). As an alternative to mGFR equations and formulas, our study suggests calculation of iGFR based on empirical Bayesian estimation of individual iohexol clearance from three-compartmental population pharmacokinetic model, similarly to software used for individualized dosing of various drugs. In addition to higher accuracy, such approach requires fewer blood samples that could be drawn at more flexible times ${ }^{42}$.

Some limitations of our study should be noted. First, we selected our patients based on eGFR values, requiring it to be [?] $80 \mathrm{~mL} / \mathrm{min} / 1.73 \mathrm{~m}^{2}$. Therefore, we might have missed some GH cases, as mGFR [?]150 $\mathrm{mL} / \mathrm{min} / 1.73 \mathrm{~m}^{2}$ has been detected in children with malignancy and eGFR $<80 \mathrm{~mL} / \mathrm{min} / 1.73 \mathrm{~m}^{2}$, but these are only few cases $3,23,48$. Second, all patients received chemotherapeutic treatment that may influence renal function ${ }^{4,5}$ within one year of the study. However, no patient received concomitant chemotherapy during the study period and previous studies have shown controversial results on its effect of GFR, some demonstrating increase after start of treatment ${ }^{4}$, others decrease ${ }^{5}$. Finally, different administrations of iohexol to one patient were considered as separate cases. However, time difference between two administrations was more than 50 days and change in patients' characteristics, such as weight, mostly large, so dependency between the measurements is negligible.

Despite high eGFR values according to eGFR equations routinely used in clinical practice, the presence of GH defined by conventionally used threshold could not be shown based on iGFR from population pharmacokinetic model of iohexol in our cohort of children and young adults with haemato-oncological disease and infection. Our data demonstrated that in such patient population eGFR equations and single- and two-point mGFR formulas overestimate GFR and are with poor precision and accuracy. Therefore, in this population renal function should be assessed by iohexol clearance from three-compartmental pharmacokinetic model, particularly in situations when renal function measurement is of utmost importance (e.g. before nephrotoxic chemotherapy or severe sepsis requiring antibacterial therapy to be within therapeutic range).

\section{Conflict of Interest statement}

The authors have no conflicts of interest to declare.

\section{Acknowledgments}

We thank all the study participants. This study was supported by the Estonian Research Council (PUT718, PUT1197, IUT34-24). The data that support the findings of this study are available from the corresponding author upon reasonable request. 


\section{References}

1. Helal I, Fick-Brosnahan GM, Reed-Gitomer B, Schrier RW. Glomerular hyperfiltration: definitions, mechanisms and clinical implications. Nat Rev Nephrol. 2012;8(5):293-300.

2. Gronroos MH, Jahnukainen T, Irjala K, et al. Comparison of glomerular function tests in children with cancer. Pediatr Nephrol.2008;23(5):797-803.

3. Jeong TD, Cho EJ, Lee W, Chun S, Hong KS, Min WK. Efficient reporting of the estimated glomerular filtration rate without height in pediatric patients with cancer. Clin Chem Lab Med. 2017;55(12):1891-1897.

4. Hjorth L, Wiebe T, Karpman D. Hyperfiltration evaluated by glomerular filtration rate at diagnosis in children with cancer. Pediatr Blood Cancer. 2011;56(5):762-766.

5. Kwatra NS, Meany HJ, Ghelani SJ, Zahavi D, Pandya N, Majd M. Glomerular hyperfiltration in children with cancer: prevalence and a hypothesis. Pediatr Radiol. 2017;47(2):221-226.

6. Van Der Heggen T, Dhont E, Peperstraete H, et al. Augmented renal clearance: a common condition in critically ill children. Pediatr Nephrol. 2019;34(6):1099-1106.

7. Llanos-Paez CC, Staatz C, Lawson R, Hennig S. Comparison of methods to estimate glomerular filtration rate in paediatric oncology patients.J Paediatr Child Health. 2018;54(2):141-147.

8. Andersen TB, Eskild-Jensen A, Frokiaer J, Brochner-Mortensen J. Measuring glomerular filtration rate in children; can cystatin C replace established methods? A review. Pediatr Nephrol.2009;24(5):929-941.

9. Blufpand HN, Tromp J, Abbink FC, et al. Cystatin C more accurately detects mildly impaired renal function than creatinine in children receiving treatment for malignancy. Pediatr Blood Cancer.2011;57(2):262-267.

10. Argyropoulos CP, Chen SS, Ng YH, et al. Rediscovering beta-2 microglobulin as a biomarker across the spectrum of kidney diseases. Front Med (Lausanne). 2017;4:73.

11. Gotoh Y, Uemura O, Ishikura K, et al. Validation of estimated glomerular filtration rate equations for Japanese children. Clin Exp Nephrol. 2018;22(4):931-937.

12. Delanaye P, Melsom T, Ebert N, et al. Iohexol plasma clearance for measuring glomerular filtration rate in clinical practice and research: a review. Part 2: Why to measure glomerular filtration rate with iohexol? Clin Kidney J. 2016;9(5):700-704.

13. Schwartz GJ, Work DF. Measurement and estimation of GFR in children and adolescents. Clin J Am Soc Nephrol. 2009;4(11):1832-1843.

14. Taubert M, Ebert N, Martus P, van der Giet M, Fuhr U, Schaeffner E. Using a three-compartment model improves the estimation of iohexol clearance to assess glomerular filtration rate. Sci Rep.2018;8(1):17723.

15. Salmon Gandonniere C, Helms J, Le Tilly O, et al. Glomerular hyper- and hypofiltration during acute circulatory failure: iohexol-based gold-standard descriptive study. Crit Care Med.2019;47(8):e623-e629.

16. Edelson J, Shaw D, Palace G. Pharmacokinetics of iohexol, a new nonionic radiocontrast agent, in humans. J Pharm Sci.1984;73(7):993-995.

17. WHO Multicentre Growth Reference Study Group. WHO Child Growth Standards based on length/height, weight and age. Acta Paediatrica. 2006;450:76-85.

18. de Onis M, Onyango AW, Borghi E, Siyam A, Nishida C, Siekmann J. Development of a WHO growth reference for school-aged children and adolescents. Bulletin of the World Health Organization.2007;85:660667.

19. Schwartz GJ, Munoz A, Schneider MF, et al. New equations to estimate GFR in children with CKD. $J$ Am Soc Nephrol. 2009;20(3):629-637. 
20. Hoste L, Dubourg L, Selistre L, et al. A new equation to estimate the glomerular filtration rate in children, adolescents and young adults. Nephrol Dial Transplant. 2014;29(5):1082-1091.

21. Bjork J, Grubb A, Sterner G, Nyman U. Revised equations for estimating glomerular filtration rate based on the Lund-Malmo Study cohort. Scand J Clin Lab Invest. 2011;71(3):232-239.

22. Gao A, Cachat F, Faouzi M, et al. Comparison of the glomerular filtration rate in children by the new revised Schwartz formula and a new generalized formula. Kidney Int. 2013;83(3):524-530.

23. Millisor VE, Roberts JK, Sun Y, et al. Derivation of New Equations to Estimate Glomerular Filtration Rate in Pediatric Oncology Patients.Pediatr Nephrol. 2017;32(9):1575-1584.

24. Brandt JR, Wong C, Jones DR, et al. Glomerular filtration rate in children with solid tumors: normative values and a new method for estimation. Pediatr Hematol Oncol. 2003;20(4):309-318.

25. Cole M, Price L, Parry A, et al. Estimation of glomerular filtration rate in paediatric cancer patients using 51CR-EDTA population pharmacokinetics. Br J Cancer. 2004;90(1):60-64.

26. Levey AS, Stevens LA, Schmid CH, et al. A new equation to estimate glomerular filtration rate. Ann Intern Med. 2009;150(9):604-612.

27. Levey AS, Coresh J, Greene T, et al. Using standardized serum creatinine values in the modification of diet in renal disease study equation for estimating glomerular filtration rate. Ann Intern Med. $2006 ; 145(4): 247-254$.

28. Cockcroft DW, Gault MH. Prediction of creatinine clearance from serum creatinine. Nephron. 1976;16(1):31-41.

29. Schwartz GJ, Schneider MF, Maier PS, et al. Improved equations estimating GFR in children with chronic kidney disease using an immunonephelometric determination of cystatin C. Kidney Int.2012;82(4):445-453.

30. Mueller L, Pruemper C. Performance in measurement of serum cystatin C by laboratories participating in the College of American Pathologists 2014 CYS Survey. Arch Pathol Lab Med. 2016;140(3):207.

31. Pottel H, Delanaye P, Schaeffner E, et al. Estimating glomerular filtration rate for the full age spectrum from serum creatinine and cystatin C. Nephrol Dial Transplant. 2017;32(3):497-507.

32. Grubb A, Horio M, Hansson LO, et al. Generation of a new cystatin C-based estimating equation for glomerular filtration rate by use of 7 assays standardized to the international calibrator. Clin Chem.2014;60(7):974-986.

33. Berg UB, Nyman U, Back R, et al. New standardized cystatin C and creatinine GFR equations in children validated with inulin clearance.Pediatr Nephrol. 2015;30(8):1317-1326.

34. Inker LA, Schmid CH, Tighiouart H, et al. Estimating Glomerular Filtration Rate from Serum Creatinine and Cystatin C. N Engl J Med. 2012;367(1):20-29.

35. Ikezumi Y, Uemura O, Nagai T, et al. Beta-2 microglobulin-based equation for estimating glomerular filtration rates in Japanese children and adolescents. Clin Exp Nephrol. 2015;19(3):450-457.

36. Inker LA, Tighiouart H, Coresh J, et al. GFR estimation using $\beta$-trace protein and $\beta 2$-microglobulin in CKD. Am J Kidney Dis.2016;67(1):40-48.

37. Tøndel C, Bolann B, Salvador CL, et al. Iohexol plasma clearance in children: validation of multiple formulas and two-point sampling times.Pediatr Nephrol. 2017;32(2):311-320.

38. Piepsz A, Tondeur M, Ham H. Revisiting normal (51)Cr-ethylenediaminetetraacetic acid clearance values in children.Eur J Nucl Med Mol Imaging. 2006;33(12):1477-1482. 
39. Bergstrand M, Karlsson MO. Handling data below the limit of quantification in mixed effect models. AAPS J.2009;11(2):371-380.

40. Krouwer JS. Why Bland-Altman plots should use $\mathrm{X}$, not $(\mathrm{Y}+\mathrm{X}) / 2$ when $\mathrm{X}$ is a reference method. Stat Med. 2008;27(5):778-780.

41. Riff C, Besombes J, Gatault P, et al. Assessment of the glomerular filtration rate (GFR) in kidney transplant recipients using Bayesian estimation of the iohexol clearance. Clin Chem Lab Med.2020;58(4):577587.

42. Åsberg A, Bjerre A, Almaas R, et al. Measured GFR by utilizing population pharmacokinetic methods to determine iohexol clearance.Kidney Int Rep. 2020;5(2):189-198.

43. Gaspari F, Perico N, Ruggenenti P, et al. Plasma clearance of nonradioactive iohexol as a measure of glomerular filtration rate.J Am Soc Nephrol. 1995;6(2):257-263.

44. Leion F, Hegbrant J, den Bakker E, et al. Estimating glomerular filtration rate (GFR) in children. The average between a cystatin C- and a creatinine-based equation improves estimation of GFR in both children and adults and enables diagnosing Shrunken Pore Syndrome. Scand J Clin Lab Invest. 2017;77(5):338-344.

45. Björk J, Nyman U, Berg U, et al. Validation of standardized creatinine and cystatin C GFR estimating equations in a large multicentre European cohort of children. Pediatr Nephrol.2019;34(6):1087-1098.

46. Pottel H, Hoste L, Dubourg L, et al. An estimated glomerular filtration rate equation for the full age spectrum. Nephrol Dial Transplant. 2016;31(5):798-806.

47. Tøndel C, Salvador CL, Hufthammer KO, et al. Iohexol plasma clearance in children: validation of multiple formulas and single-point sampling times. Pediatr Nephrol. 2018;33(4):683-696.

48. Bernhardt MB, Moffett BS, Johnson M, Tam VH, Thompson P, Garey KW. Agreement among measurements and estimations of glomerular filtration in children with cancer. Pediatr Blood Cancer. 2015;62(1):8084.

\section{Legends}

FIGURE 1 Observed versus population (left panel) and individual (right panel) predicted concentrations from the final population pharmacokinetic model of iohexol.

\section{Hosted file}

Table1.pdf available at https://authorea.com/users/394534/articles/507907-glomerularfiltration-rate-in-children-and-young-adults-with-haemato-oncological-disease-andinfection-is-best-described-by-three-compartmental-iohexol-model

\section{Hosted file}

Table2.pdf available at https://authorea.com/users/394534/articles/507907-glomerularfiltration-rate-in-children-and-young-adults-with-haemato-oncological-disease-andinfection-is-best-described-by-three-compartmental-iohexol-model

\section{Hosted file}

Table3.pdf available at https://authorea.com/users/394534/articles/507907-glomerularfiltration-rate-in-children-and-young-adults-with-haemato-oncological-disease-andinfection-is-best-described-by-three-compartmental-iohexol-model

\section{Hosted file}

Table4.pdf available at https://authorea.com/users/394534/articles/507907-glomerularfiltration-rate-in-children-and-young-adults-with-haemato-oncological-disease-andinfection-is-best-described-by-three-compartmental-iohexol-model 

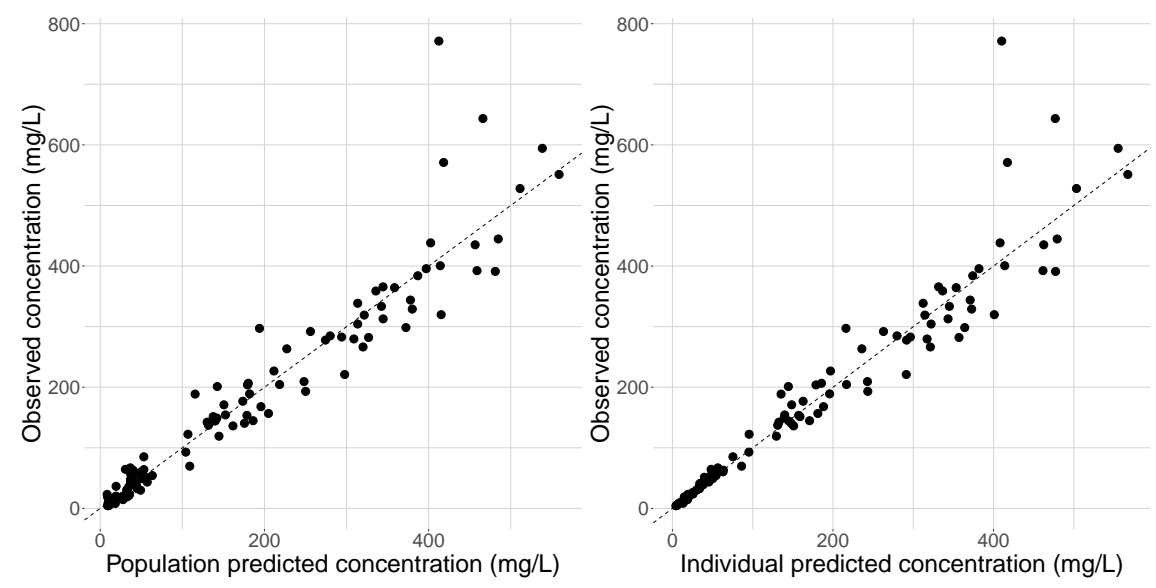